Check for updates

Cite this: RSC Adv., 2017, 7, 39299

\title{
Surfaces enhanced with film-coupled silver nanopolyhedrons for optical transmittance $\uparrow$
}

\author{
Xiuhui Bai, (D) a Jigang Wang, ${ }^{a}$ Ming Guo, ${ }^{a}$ Zhen Li, ${ }^{a}$ Ning Chen, ${ }^{a}$ Qiang Wang, (D) *a \\ Chunhong Li, ${ }^{b}$ Changzheng Wang, (D) *c Kun Dong ${ }^{c}$ and Shaowei Chen (iD *d
}

A metallic nanoparticle positioned over a metal film offers enormous advantages as a highly controllable system relevant for probing field-enhancement and designing controlled-emissivity surfaces for thermophotovoltaic devices. The film-coupled silver (Ag) nanoparticle is of particular interest due to the formation of waveguide cavity-like modes between the NPs and film. The ability of individual nanopolyhedron (NP) patch antennas, consisting of Ag NP separated from gold (Au) film by a dielectric spacer layer spacer, to act as efficient and tunable absorption elements is demonstrated. The size and shape of the gap between the nanoparticle and film can be precisely controlled using relatively simple, bottom-up fabrication approaches. We show that the film-coupled NPs provide a transmission spectrum that can be tailored by varying the geometry (the size of the NPs and/or the thickness of the spacer). We perform both experimental spectroscopy and numerical simulations of individual NPs positioned over Au film, finding excellent agreement between experiment and simulation. The waveguide mode description serves as a starting point to explain the optical properties observed.

Received 6th May 2017

Accepted 4th August 2017

DOI: $10.1039 / \mathrm{c} 7 \mathrm{ra0} 5126 \mathrm{c}$

rsc.li/rsc-advances

\section{Introduction}

Noble-metal nanocrystals have been extensively studied in recent years due to their remarkable physicochemical properties, which are substantially different from those of bulk materials. ${ }^{1-7}$ Controlled synthesis of noble-metal nanoparticles in terms of size and shape has been strongly motivated by the requirements to uncover and map their size- and shapedependent properties and to achieve their practical applications. ${ }^{8-12}$ Silver $(\mathrm{Ag})$, in particular, a noble metal historically used in the fabrication of ornaments, electrical conductors, mirrors, antimicrobial coatings, and photographic films ${ }^{13-17}$ has received ever increasing attention when it is in the form of nanocrystals because of their superior performance in a broad range of applications such as catalysis, electronics, optical labeling, and biosensing. ${ }^{18-22}$ Even more remarkably, over the last few years there has been a surge in the interest for surface

${ }^{a}$ Laboratory for Micro-sized Functional Materials \& College of Elementary Education, Department of Chemistry, Capital Normal University, Beijing, 100048, P. R. China. E-mail: qwchem@gmail.com

${ }^{b}$ Beijing National Laboratory for Condensed Matter Physics, Institute of Physics, Chinese Academy of Sciences, P.O. Box 603, Beijing 100190, China

'Key Laboratory of Urban Stormwater System and Water Environment, Ministry of Education, Beijing University of Civil Engineering and Architecture, Beijing, 100044, P. R. China. E-mail: changzhwang@163.com

${ }^{d}$ Department of Chemistry and Biochemistry, University of California, Santa Cruz, CA 95064, USA.E-mail: shaowei@ucsc.edu

$\dagger$ Electronic supplementary information (ESI) available. See DOI: $10.1039 / \mathrm{c} 7 \mathrm{ra05126c}$ plasmon optics. The extremely strong and localized electromagnetic fields generated in such silver systems open fascinating applications in enhancing molecular spectroscopies, ${ }^{23,24}$ refractive index sensing, ${ }^{25,26}$ and nonlinear optics processing at the nanoscale. ${ }^{27-29}$ Unfortunately, isolated nanoparticles cannot typically achieve these properties, yet when multiple plasmonic structures are coupled, tunable optical properties for precise deployment across a wide range of the electromagnetic spectrum become possible..$^{30-37}$

Confronted with the above-mentioned problems, coupled plasmonic nanostructures, controlling and tuning the optical properties though precisely manipulating the gap size between them, have been studied extensively. To date, the experimental study of coupled plasmonic systems has been hampered by the difficulty in achieving reliable and precise control of subnanometer interparticle spacing. Even a relatively simple system, such as two nanospheres separated by a subnanometer gap, remains a challenge to colloidal or lithographic synthesis methods. By contrast, the system of a metallic nanoparticle positioned over a metallic film represents an alternative class of coupled plasmonic structures that sidesteps the fabrication difficulties associated with the methods described above. The size and shape of the gap between the nanoparticle and film can be controlled to subnanometer precision using relatively simple, bottom-up fabrication approaches. As the nanoparticles (NPs) are brought closer to the film, the coupling between a given NP and its virtual image could also enhance optical fields. When light impinges on the NP-film structure, resonant oscillations of the conduction electrons, known as surface 
plasmons, are excited at which the elastic scattering cross section of the particle increases dramatically and the local electromagnetic fields are strongly enhanced. Although they have dimensions smaller than the diffraction limit of light, plasmon resonant NPs can, nevertheless, be readily observed individually in a microscope under appropriate illumination conditions where background scattering is minimized. ${ }^{\mathbf{3 8 - 4 0}}$

In real life, efficient and tunable absorption is essential for a variety of applications. It has been discovered that the NP-film system, made reflectionless for light incident over a broad range of angles, can be regard as an ideal absorber. ${ }^{41-43}$ Similarly, whether this model can also affect the transmittance? To this day, enormous effort has been made for the NP-film system and, for this matter, is continuing. However, most of them are particular interest in the film-coupled nanocube or nanosphere and scattering spectrum. ${ }^{22,30,38,39}$ As we all known, nanopolyhedron has much edges than nanocube. ${ }^{44-46}$ Whether the film-coupled nanopolyhedron will have a better performance, and whether this model can also affect the transmittance? There is no systematic study or discussion of the above issues according to the available literature.

The relationship between the transmittance coefficient $\tau^{(n)}$ and reflection coefficient $r_{\mathrm{s}}$ can be described by eqn (1),

$$
\tau^{(n)}=\frac{1-r_{\mathrm{s}}}{1+(2 n-1) r_{\mathrm{s}}} \tau_{\mathrm{a}}{ }^{n}
$$

where $n$ the number of objects, and this is the approximate expression of Hottel and Woertz. What's more, the above formula is deduced by ignoring the absorption of reflected light. For a material defined by its electric permittivity, $\varepsilon$, and magnetic permeability, $\mu$, the Fresnel reflection coefficient for the $s$ polarization has the form described by eqn (2),,$^{30}$

$$
r_{\mathrm{s}}=\frac{z_{2} \cos \theta_{\mathrm{i}}-z_{1} \cos \theta_{\mathrm{t}}}{z_{2} \cos \theta_{\mathrm{i}}+z_{1} \cos \theta_{\mathrm{t}}}
$$

where $z_{j}=\sqrt{\mu_{j} / \varepsilon_{j}}$ is the wave impedance for material $j$, and $\theta_{\mathrm{i}}$ and $\theta_{\mathrm{t}}$ are the incident and transmitted angle of the wave with respect to the surface normal. For normal incidence, for which $\theta_{\mathrm{i}}=\theta_{\mathrm{t}}=0$, eqn (2) shows that when $z_{1}=z_{2}$ the reflection is eliminated. For normal incidence, any medium whose wave impedance has the vacuum value will be reflectionless in the vacuum.

We view the surface as supporting both electric as well as fictitious magnetic surface current densities that are excited by the incident wave. Because both currents produce reflected waves that are exactly out of phase with each other, their sum can cancel out entirely if the total induced effective magnetic current exactly balances the total induced electric current. The film-coupled NPs support a series of cavity-like resonances in which the electromagnetic field is localized within the gap between the ground plane and the patch element. For modes in which the electric field is maximum at the patch edges, the patch can be equivalently described as having a magnetic surface current density, $K_{\mathrm{M}}$, that flows along the periphery of the patch, with magnitude given by $2 E \times n$, where the field is determined at the patch edge and $n$ is a unit vector normal to the surface. Thus, changing the population or size of NPs and the thickness of the spacer layer can all generate different fictitious magnetic surface current density to offset the electric surface current density of an incident wave, achieving the level of control needed in certain spectroscopy and energy-harvesting applications.

Having evaluated the underlying mechanisms of the nanoparticle based model, we next seek to implement the concept model. Here we demonstrate a simple method to create a NP-film model by randomly adsorbing chemically synthesized $\mathrm{Ag}$ NPs onto a nanoscale-thick polymer spacer layer on an $\mathrm{Au}$ film, making no effort to control the spatial arrangement of the $\mathrm{Ag}$ NPs on the film. As expected, we discovered that the filmcoupled NPs provide a transmission spectrum that can be tailored by varying the size of the Ag NPs, the thickness of the spacer or the coverage density.

\section{Experimental}

\section{Materials}

Ethylene glycol (A.R.), PVP $\left(M_{\mathrm{r}} \approx 58000\right), \mathrm{NaCl}(\geq 99.8 \%$, A.R.), $\mathrm{AgNO}_{3}$ ( $\geq 99.8 \%$, A.R.), and $\mathrm{Zn}(\geq 90 \%$, A.R.). All of the materials and reagents were used directly without further purification. Distilled water was purified with a Milli-Q system (Millipore, Billerica, MA). A stirring hotplate with a temperature controller (Ms7-H550-Pro, Dragon Laboratory Instruments limited), and micropipettes (Eppendorf Research ${ }^{\circledR}$ Plus) were used.

\section{Synthesis of silver nanopolyhedrons}

Silver nanopolyhedrons were chemically synthesized by a further improved polyol approach in EG-based polyol system with zinc as the additive or promoter at $140{ }^{\circ} \mathrm{C}$ (Fig. S1-S4†). In a typical synthesis, $5 \mathrm{ml}$ of ethylene glycol was held in a $20 \mathrm{ml}$ glass vial and heated in an oil bath at $140{ }^{\circ} \mathrm{C}$ under magnetic stirring for $1 \mathrm{~h}$. $\mathrm{NaCl}$ ( $1 \mathrm{ml}$ of $0.25 \mathrm{mM}$ in EG) was then quickly added. After about 10 minutes, suspension of zinc $(60 \mu \mathrm{l}$ of $0.09 \mathrm{~g}$ in $5 \mathrm{ml} \mathrm{EG}$ ) was added to the solution. After at least $5 \mathrm{~min}$, $\mathrm{AgNO}_{3}$ (3 $\mathrm{ml}$ of a $99.13 \mathrm{mM}$ solution in EG) and poly(vinyl pyrrolidone) (PVP, $3 \mathrm{ml}$ of a solution in EG (312 $\mathrm{mM}$ in terms of the repeating unit, $\left.M_{\mathrm{r}} \approx 58000\right)$ ) were simultaneously added to the stirring solution. Once the reaction was completed (solution was a celadon color), the glass vial was removed from the hot oil and placed in an ice bath to cool. The contents of the glass vial were transferred to a centrifuge tube, rinsed once with acetone to remove any remaining organics, and centrifuged at $12000 \mathrm{rpm}$ for $10 \mathrm{~min}$. The supernatant was removed, and the nanopolyhedron solution was resuspended in deionized water adequately. The solution was then centrifuged at $10000 \mathrm{rpm}$ for $4 \mathrm{~min}$, and the supernatant was again removed. The centrifugal process is repeated four times. The addition of the PVP during the reaction results in a thin layer of PVP coating the nanopolyhedrons, which we estimate to be about $2 \mathrm{~nm}$.

\section{Construction of surface enhanced film-coupled Ag nanopolyhedrons}

Substrates were prepared by sputtering a $50 \mathrm{~nm} \mathrm{Au}$ film with a $5 \mathrm{~nm}$ Cr adhesion layer onto a Nexterion Glass B slide via 
magnetron sputtering apparatus (MSP-620). LBL deposition of poly(allylamine) hydrochloride ( $\mathrm{PAH}, M_{\mathrm{w}}=70 \mathrm{kDa}$, Aldrich) and polystyrene sulfonate (PSS, $M_{\mathrm{w}}=70 \mathrm{kDa}$, Aldrich) was used to create dielectric spacers to controllably separate gold NPs from gold films. For each adsorption step the gold-coated slides were immersed in 0.003 moles-of-monomer per L (monomol per L) polyelectrolyte and $1 \mathrm{M} \mathrm{NaCl}$ for $30 \mathrm{~min}$, rinsed thoroughly with a gentle stream of ultra-pure water ( $18 \mathrm{M} \Omega$, used in all experiments), and immersed in fresh ultra-pure water for $1 \mathrm{~min}$, after which the substrates were either immersed in $1 \mathrm{M} \mathrm{NaCl}$ for $30 \mathrm{~s}$ before repeating the same steps for deposition of the oppositely charged PE or dried with a stream of nitrogen for analysis.

\section{Characterization methods}

The size distribution and morphology of the $\mathrm{Ag}$ nanopolyhedrons were examined by scanning microscopy (SEM, HITACHI, S4800, $15 \mathrm{kV}$ ) studies. The samples for SEM were then prepared by drop-casting the ethanol solution containing $\mathrm{Ag}$ NPs and EG on a Si substrate that had been patterned with registration marks and letting it dry under ambient conditions. The Ultraviolet-Visible (UV-Vis) spectra extinction spectrum for the solution diluted six times with ethanol was obtained at room temperature on a Lambda $750 \mathrm{~S}$ spectrometer (PerkinElmer). Experimental transmittance measurements with the resolution of $0.1 \mathrm{~nm}$ were performed on a SP2555 grating spectrophotometer with a detector and a photomultiplier tube. The Raman spectroscopy was obtained by the Renishaw inVia Raman microscope with $633 \mathrm{~nm}$ laser wavelength.

\section{Results and discussion}

In order to prove whether the poly(allylamine) hydrochloride (PAH) and polystyrene sulfonate (PSS) have been coated on the gold film, Raman spectra of the nanoantennas were measured using $633 \mathrm{~nm}$ wavelength laser. According to the Raman spectra (Fig. 1), we find the positive results. There is a strong absorption peak at $900 \mathrm{~cm}^{-1}$ in the Raman spectra, which is caused by the breathing vibration of benzene ring. In addition, the peak at $1590 \mathrm{~cm}^{-1}$ is resulting from the stretching vibration of $\mathrm{C}-\mathrm{N}$, bending vibration of $\mathrm{N}-\mathrm{H}$ and asymmetric stretching vibration of carbon atoms in the benzene ring. The telescopic absorption peak of sulfonic acid

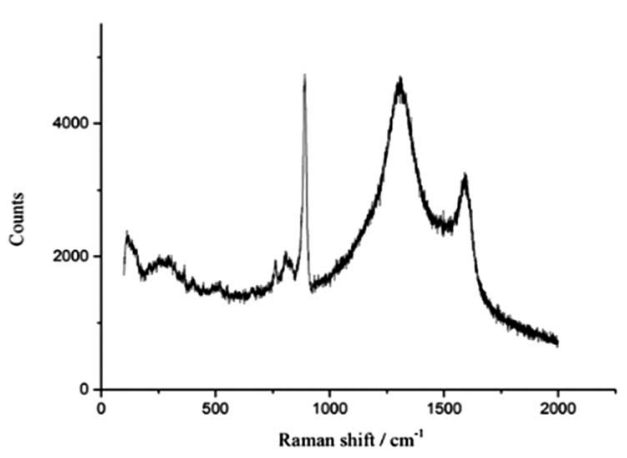

Fig. 1 Raman spectra of the NPs-film system. group is shown at $1319 \mathrm{~cm}^{-1}$. So, in summary, there is no doubt the PAH and PSS have been coated on the gold film.

The nanopolyhedrons were immobilized on the polyelectrolyte surface by brief exposure to the colloidal solution followed by rinsing with water and drying under a stream of nitrogen. In an effort to limit the number of aggregates accumulating on the surface of the film during this attachment process, the nanopolyhedrons solution was sonicated briefly and the slide was lowered gold side down onto a coverslip containing a $10 \mathrm{ml}$ droplet of the colloidal solution, whereupon the coverslip was picked up by the slide using the capillary force of the droplet spreading onto the film surface. Under these conditions, aggregates would theoretically sink away from the surface of the gold film during the incubation. The density of coverage of nanopolyhedrons on the surface of the gold film was controlled by varying the concentration of colloids in solution and the time that the surface was exposed to the colloidal solution. Scanning electron microscope images were used to determine the surface density of the nanopolyhedrons and their size distribution. The films were rinsed with ultrapure water and dried with nitrogen. The geometry of the final Ag nano-overmetal film system is shown in Fig. 2 .

Herein we report a further improved polyol approach to obtain monodispersed Ag NPs in an EG-based polyol system with zinc as the additive or promoter at $140{ }^{\circ} \mathrm{C}$. We demonstrate that we can drastically improve the production rate of silver NPs by adding a trace amount of zinc. The size distribution of the Ag NPs can be estimated from the SEM images (Fig. 3A). Fig. 3B shows the statistical distribution of the size.

Optical transmittance experiments were performed on a SP2555 grating spectrophotometer with a detector, a photomultiplier tube and the resolution of $0.1 \mathrm{~nm}$. Transmission spectra were obtained from samples where about $15 \%$ of the surface of the gold film was occupied by silver NPs (Fig. 4A). All transmission spectra were background corrected by subtracting the spectrum from an aperture region of the substrate with no NPs and normalized to correct for the wavelength response of the apparatus by dividing with the scattered spectrum from a white scattering standard. We could have modulated the NPs-film separation distance by increasing polyelectrolyte molecular spacer layers assuming that the NPs-film separation distance is the sum of the polyelectrolyte spacer layer thickness.

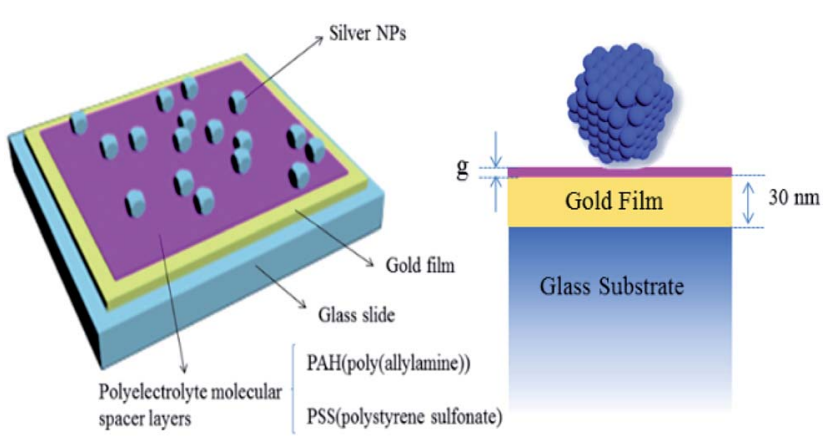

Fig. 2 Schematic illustration of plasmon mode of film-coupled Ag nanopolyhedrons. 

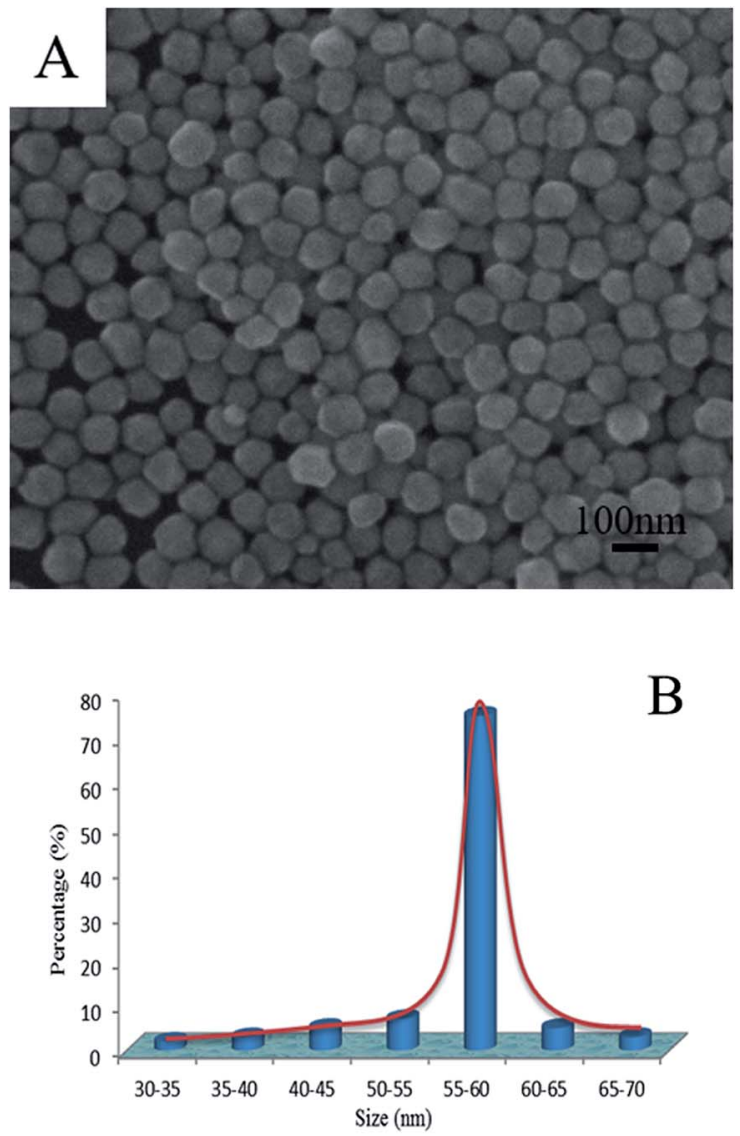

Fig. 3 SEM image of the silver NPs as fabricated (A) and sizes distribution of the NPs (B).

Transmittance spectra were measured at different polyelectrolyte (PE) molecular spacer layers (Fig. 4B). For each one of the NP-film samples has different dielectric thickness, but for all of them the surface coverage with the same density (15\%) of Ag NPs. The size dispersion can be taken into account by considering that the absorption by the surface is a statistical average of the absorption by NPs with different sizes. Two absorption peaks were observed in the transmittance spectra. The peak at $430 \mathrm{~nm}$ is the characteristic absorption peak of silver NPs, not changing with the different polyelectrolyte (PE) molecular spacer layers. It should be noted that there are dynamic peaks, corresponding to the relative change of the transmittance. Due to the inverse relationship between the mode effective index and spacer thickness, scattering from NPs above thinner films will show a much greater shift in resonance wavelength than thicker films for a given change in spacer thickness (ESI $\dagger$ ). The same phenomenon also appears in through the transmission spectrum. As shown in the Fig. 4B, it can be concluded that the $T \%$ of the samples will mostly decrease with the increasing number of PE layers. A transmittance of less than $49 \%$ is observed for normal incidence at $605 \mathrm{~nm}$ from the sample, further demonstrating the ease and extent to which the transmittance can be controlled. It's not the larger the better of the thickness, however, the absorption peak will

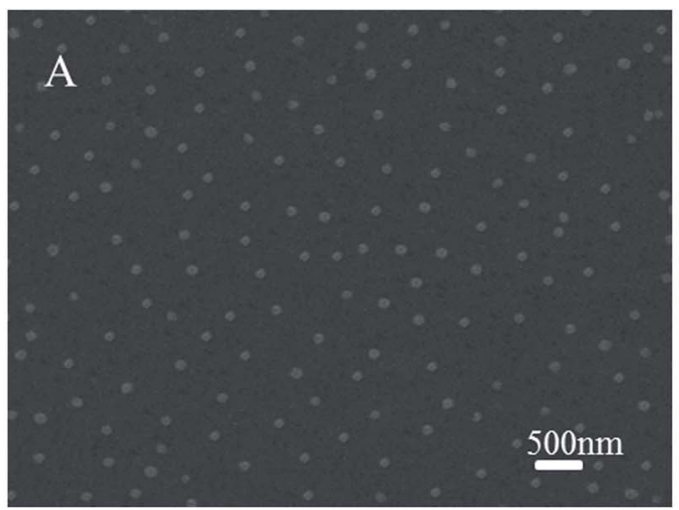

B

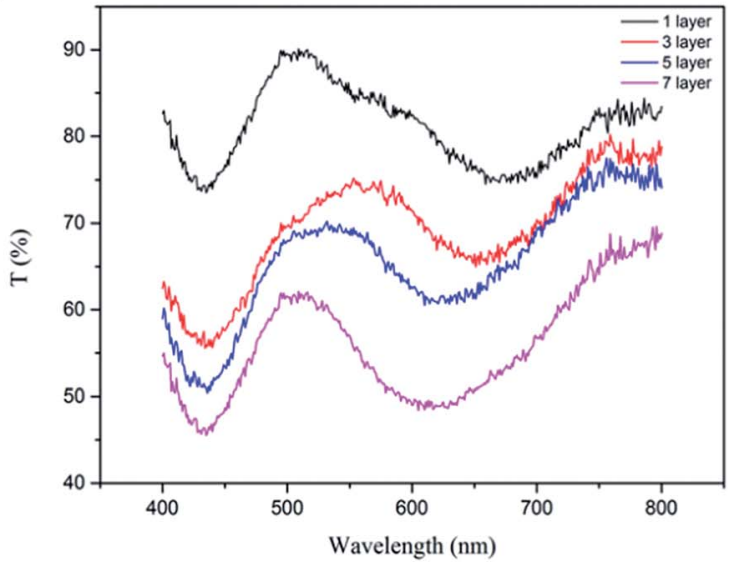

C

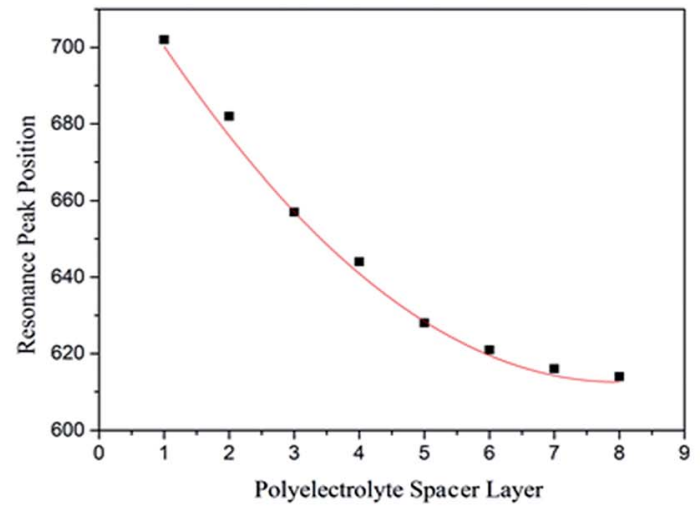

Fig. 4 (A) SEM images of the silver NPs deposited on the gold film (B) tunability of the transmittance corresponding to different spacer thickness (C) measured positions (black squares) for a certain silver NPs surface density agree with three-dimensional simulations (red line) for silver NPs surrounded by air.

become smooth if the thickness is too larger or too thinner. If the distance is too far, the silver nanoparticle couldn't form effective mirror images ${ }^{30,40}$ on gold (acts as a mirror). So the spectral response sensitivity is reduced. However, for many applications, a narrow absorption band may be desirable. Therefore, the polymer layer should be no more than 6 layers. In order to get a sensitive response. 
Fig. 4C shows the position of the measured resonance as a function of the overall thickness under the nanopolyhedrons, assuming that the nanopolyhedrons-film separation distance is the sum of the polyelectrolyte spacer layer thickness and the $2 \mathrm{~nm}$ thickness of the stabilizer coating that surrounds the nanopolyhedrons. The position of the resonances can be accurately determined by three-dimensional simulations if the coating and the rounded corners of the NPs are taken into account (by using a somewhat smaller nanopolyhedrons dimension of $60 \mathrm{~nm}$ ). As shown in the Fig. 4C, a clear reciprocal relationship between thickness and resonance wavelength is observed, with a stronger blue shifting of the peak for thinner films in line with an increasing dielectric thickness. As well as expected, this trend is consistent with three-dimensional simulations for silver NPs surrounded by air. The resonance peak is very sensitivity to spacing between the NP and the film from 1 to 6 layers with the resonance peak shift $13.2 \mathrm{~nm}$ per layer increasing. It is not difficult to find that the curve of resonance peak changes with the increase of dielectric
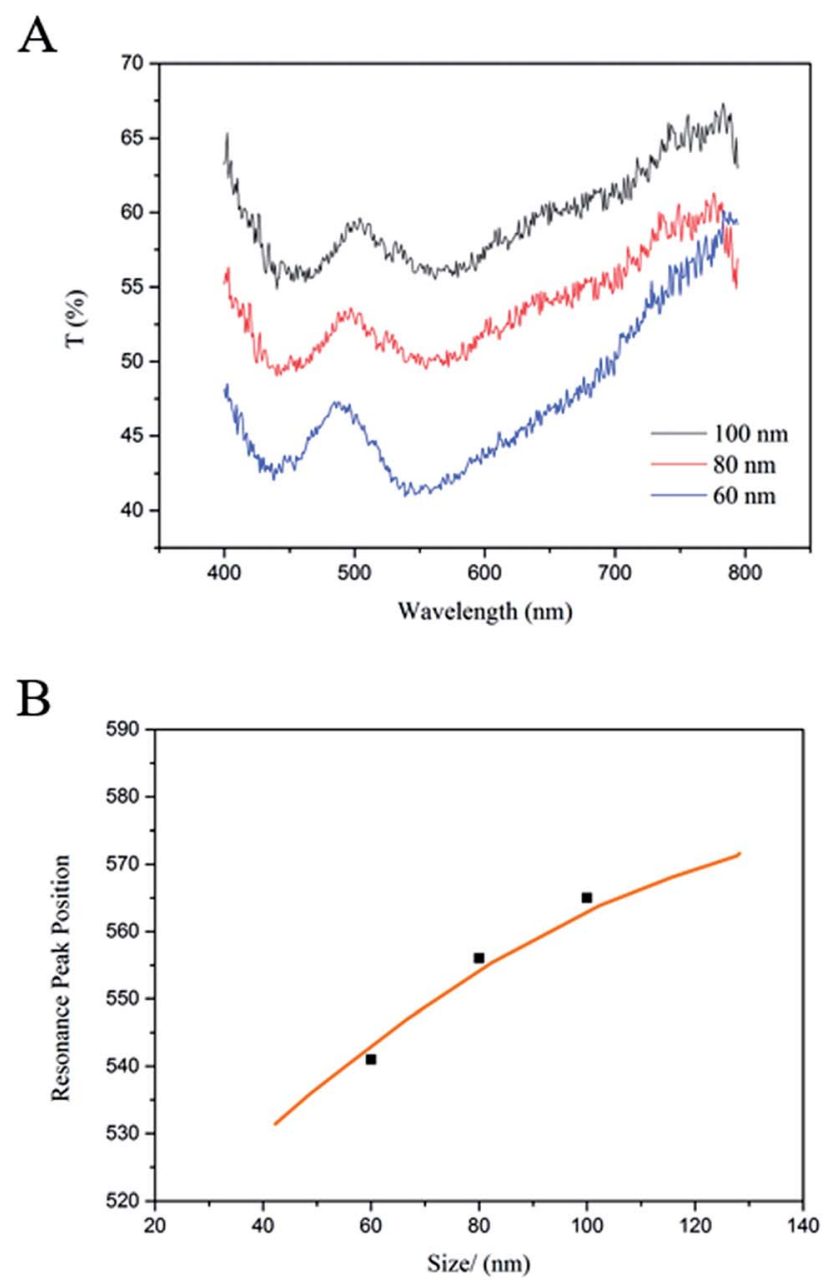

Fig. 5 (A) Tunability of the transmittance corresponding to different size of Ag nanopolyhedrons with a layer-by-layer (LBL) deposition of polyelectrolyte (PE) layers of three (B) measured positions (black squares) for a certain silver NPs surface density (15\%) agree with threedimensional simulations (red line) for silver NPs surrounded by air. thickness, and becomes more and more flat. With the dielectric thickness increasing, the silver particle is away from the metal film, the surface plasmon polaritons of the gold film could not be preferentially excited, and therefore, the localized plasmon resonance of the silver nanoparticle dominates the spectra. In addition, the position of the resonance is not significantly sensitive to $\mathrm{P}$ polarization or $\mathrm{S}$ polarization.

Since different sizes lead to different resonance frequencies, a surface covered with NPs of different sizes leads to different transmittance spectrum. The size distribution of the silver NPs can be estimated from the SEM images (Fig. S5 $\dagger$ ). Fig. 5A shows the corresponding experimental transmittance for the different size keeping all the other geometrical parameters constant. The thickness between gold film and nanopolyhedrons is three spacer layers and the surface density is $15 \%$. As shown in Fig. 5A, it can be concluded that the $T \%$ of the samples will mostly decrease with the size decreasing of silver NPs, and which is exactly what were looking for. More importantly, the absorption peaks become sharper with the size decreasing, which is always desirable for a narrow absorption band in many optics application fields. ${ }^{47,48}$ Fig. 5B shows the position of the measured resonance as a function of the nanoparticle scale. Resonance peak positions (black squares) for a certain silver NPs surface density (15\%) are measured, which are agree with three-dimensional simulations (orange line) for silver NPs surrounded by air.

In addition to sizes and spacer thicknesses, the surface coverage of $\mathrm{Au}$ film also influences the results of the transmittance, which is shown in Fig. 6 (Fig. S7†). In this part, the surface coverage density is varied while all the other geometrical parameters remains constant. The thickness between gold film and nanopolyhedrons is four spacer layers and the nanopolyhedrons size is $60 \mathrm{~nm}$. Theoretically, the concentration of Ag NPs deposited on the film surface was intentionally kept low in order to facilitate single NPs characterization and to minimize both interparticle LSP coupling and shifts to the gold film SPPs, which can be induced by high concentrations of any surface defect. When the surface density is very low, the spacing between nanoparticles is greater than the incident wavelength,

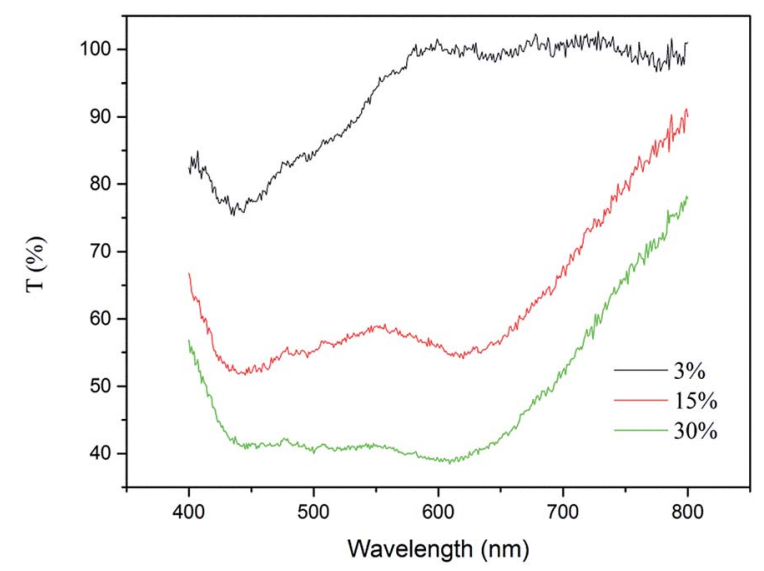

Fig. 6 Tunability of the transmittance corresponding to different surface coverage. 
which leads to no absorption around $600 \mathrm{~nm}$. Similarly, the high concentrations will result in both interparticle LSP coupling and shifts to the gold film SPPs.

\section{Conclusions}

In conclusion, we have fabricated $\mathrm{Ag}$ NPs, constructed the device of surfaces enhanced film-coupled Ag nanopolyhedrons and measured optical transmittance for the device. The bottomup, colloidal fabrication approach described here is simple, rapid, inexpensive and easily scalable, and could lead to a robust method to experimentally control a plasmonic junction with extreme precision. According to detailed experimental characterization, we found that the observed resonances can be understood as waveguide cavity modes, where the gap between the Ag NPs and the metal film defines a waveguide cavity where electromagnetic energy can resonantly reflect back and forth. The transmission spectra are strongly dependent on the gap size separating the NPs from the metal film, a property that could potentially be exploited for active tuning applications. The transmission spectra are also dependent on the size of the NPs and the surface cover density, offering a straightforward method for tuning the optical resonances over a broad range. The spectral control available from absorbing metasurfaces forms the basis for a growing number of promising applications, including thermal detectors, light sources, energyharvesting systems and even biosensors.

\section{Acknowledgements}

This work was supported by the Natural Science Foundation of China (NSFC, No. 21471103, 11574173) and the Scientific Research Base Development Program of the Beijing Municipal Commission of Education.

\section{Notes and references}

1 S. Zhou, J. Li, K. D. Gilroy, J. Tao, C. Zhu, X. Yang, X. Sun and Y. Xia, ACS Nano, 2016, 10, 9861.

2 Y. Xia, K. D. Gilroy, H. C. Peng and X. Xia, Angew. Chem., Int. Ed., 2017, 56, 60.

3 A. Sánchez-Iglesias, N. Winckelmans, T. Altantzis, S. Bals, M. Grzelczak and L. M. Liz-Marán, J. Am. Chem. Soc., 2017, 139, 107.

4 Y. Wang, Y. Zheng, C. H. Huang and Y. Xia, J. Am. Chem. Soc., 2013, 135, 1941.

5 Q. Zhang, W. Li, C. Moran, J. Zeng, J. Chen, L. P. Wen and Y. Xia, J. Am. Chem. Soc., 2010, 132, 11372.

6 J. N. Anker, W. P. Hall, O. Lyandres, N. C. Shah, J. Zhao and R. P. Van Duyne, Nat. Mater., 2008, 7, 442.

7 Y. Xia, Y. Xiong, B. K. Lim and S. E. Skrabalak, Angew. Chem., Int. Ed., 2009, 48, 60.

8 D. V. Talapin, J. S. Lee, M. V. Kovalenko and E. V. Shevchenko, Chem. Rev., 2010, 110, 389.

9 S. E. Skrabalak, L. Au, X. Li and Y. Xia, Nat. Protoc., 2007, 2, 2182.

10 A. Ruditskiy and Y. Xia, J. Am. Chem. Soc., 2016, 138, 3161.
11 P. Christopher and S. Linic, ChemCatChem, 2010, 2, 78.

12 L. Pourzahedi and M. J. Eckelman, Environ. Sci. Technol., 2015, 49, 361.

13 M. Rycenga, C. M. Cobley, J. Zeng, W. Li, C. H. Moran, Q. Zhang, D. Qin and Y. Xia, Chem. Rev., 2011, 111, 3669.

14 S. Nie and S. R. Emory, Science, 1997, 275, 1102.

15 X. Xia, J. Zeng, B. M. Dearmon, Y. Zheng, Q. Li and Y. Xia, Angew. Chem., Int. Ed., 2011, 50, 12542.

16 K. Aslan, M. Wu, J. R. Lakowicz and C. D. Geddes, J. Am. Chem. Soc., 2007, 129, 1524.

17 A. Kumar, P. K. Vemula, P. M. Ajayan and G. John, Nat. Mater., 2008, 7, 236.

18 J. He, I. Ichinose, T. Kunitake, A. Nakao, Y. Shiraishi and N. Toshima, J. Am. Chem. Soc., 2003, 125, 11034.

19 Y. Wu, Y. Li and B. S. Ong, J. Am. Chem. Soc., 2007, 129, 1862. 20 A. M. Schrand, L. K. Braydich-Stolle, J. J. Schlager, L. Dai and S. M. Hussain, Nanotechnology, 2008, 19, 235104.

21 H. Peng, C. M. Strohsahl, K. E. Leach, T. D. Krauss and B. L. Miller, ACS Nano, 2009, 3, 2265.

22 J. Zhang, Q. Wang, X. Zhang, J. Wang, M. Guo, B. J. Wiley, C. Li and C. Hu, Inorg. Chem. Front., 2016, 3, 547.

23 A. Rose, T. B. Hoang, F. McGuire, J. J. Mock, C. Ciraci, D. R. Smith and M. H. Mikkelsen, Nano Lett., 2014, 14, 4797.

24 K. Kneipp, Y. Wang, H. Kneipp, L. T. Perelman, I. Itzkan, R. R. Dasari and M. S. Feld, Phys. Rev. Lett., 1997, 78, 1667.

25 L. J. Sherry, S. Chang, G. C. Schatz and R. P. V. Duyne, Nano Lett., 2005, 5, 2034.

26 N. Liu, M. Mesch, T. Weiss, M. Hentschel and H. Giessen, Nano Lett., 2010, 10, 2342.

27 M. Danckwerts and L. Novotny, Phys. Rev. Lett., 2007, 98, 026104.

28 S. Kim, J. Jin, Y. Kim, I. Park, Y. Kim and S. Kim, Nature, 2008, 453, 757.

29 L. Yang, H. Wang, Y. Fang and Z. Li, ACS Nano, 2016, 10, 1580.

30 M. Antoine, C. Ciracì, J. J. Mock, R. T. Hill, Q. Wang, B. J. Wiley, A. Chilkoti and D. R. Smith, Nature, 2012, 492, 86.

31 S. Schultz, D. R. Smith, J. J. Mock and D. A. Schultz, Proc. Natl. Acad. Sci. U. S. A., 2000, 97, 996.

32 G. Raschke, S. Kowarik, T. Franzl, C. Sonnichsen, T. A. Klar and J. Feldmann, Nano Lett., 2003, 3, 935.

33 Y. Yang, Y. H. Lee, I. Y. Phang, R. Jiang, H. Y. F. Sim, J. Wang and X. Y. Ling, Nano Lett., 2016, 16, 3872.

34 M. S. Tame, K. R. McEnery, S. K. Özdemir, J. Lee, S. A. Maier and M. S. Kim, Nat. Phys., 2013, 9, 329.

35 J. M. McMahon, S. K. Gray and G. C. Schatz, Nano Lett., 2010, 10, 3473.

36 C. David and F. J. Abajo, J. Phys. Chem. C, 2011, 115, 19470. 37 T. V. Teperik, P. Nordlander, J. Aizpurua and A. G. Borisov, Opt. Express, 2013, 21, 27306.

38 J. B. Lassiter, F. McGuire, J. J. Mock, C. Ciracì, R. T. Hill, B. J. Wiley, A. Chilkoti and D. R. Smith, Nano Lett., 2013, 13, 5866.

39 C. Ciraci, R. T. Hill, J. J. Mock, Y. Urzhumov, A. I. FernándezDomínguez, S. A. Maier, J. B. Pendry, A. Chilkoti and D. R. Smith, Science, 2012, 337, 1072. 
40 J. J. Mock, R. T. Hill, A. Degiron, S. Zauscher, A. Chilkoti and D. R. Smith, Nano Lett., 2008, 8, 2245.

41 N. I. Landy, S. Sajuyigbe, J. J. Mock, D. R. Smith and W. J. Padilla, Phys. Rev. Lett., 2008, 100, 207402.

42 J. Hao, J. Wang, X. Liu, W. J. Padilla, L. Zhou and M. Qiu, Appl. Phys. Lett., 2010, 96, 251104.

43 X. Liu, T. Tyler, T. Starr, A. F. Starr, N. M. Jokerst and W. J. Padilla, Phys. Rev. Lett., 2011, 107, 045901.

44 B. J. Wiley, Y. Sun and Y. Xia, Acc. Chem. Res., 2007, 40, 1067.
45 R. Long, S. Zhou, B. J. Wiley and Y. Xiong, Chem. Soc. Rev., 2014, 43, 6288.

46 X. Lu, M. Rycenga, S. E. Skrabalak, B. J. Wiley and Y. Xia, Annu. Rev. Phys. Chem., 2009, 60, 167.

47 C. Koechlin, P. Bouchon, F. Pardo, J. Jaeck, X. Lafosse, J. Pelouard and R. Haïdar, Appl. Phys. Lett., 2011, 99, 241104.

48 X. Liu, T. Tyler, T. Starr, A. F. Starr, N. M. Jokerst and W. J. Padilla, Phys. Rev. Lett., 2011, 107, 045901. 